\title{
Antibacterial Efficacy and Cytotoxicity of Silver-Nanoparticle-Based Coatings Facilitated by a Plasma Deposited Polymer Interlayer
}

\author{
Shima Taheri, ${ }^{a}$ Alex Cavallaro, ${ }^{\text {a }}$ Mary Barton, ${ }^{\mathrm{c}}$ Jason D Whittle, ${ }^{\mathrm{a}}$ \\ Peter Majewski, ${ }^{a}$ Louise E Smith, ${ }^{b}$ and Krasimir Vasilev ${ }^{a, b^{*}}$ \\ aSchool of Engineering, University of South Australia, Mawson Lakes, SA 5095 Australia; ${ }^{\text {Maw- }}$ \\ son Institute, University of South Australia, Mawson Lakes, SA 5095 Australia; 'School of Phar- \\ macy and Medical Sciences, University of South Australia, SA 5000, Australia
}

*Address correspondence to: Krasimir Vasilev, Mawson Institute, Mawson Lakes Campus, Mawson Lakes, SA 5095 Australia; Tel.: +61-8-83025697; Fax:+61-8-83025689; Email: krasimir.vasilev@unisa.edu.au

\begin{abstract}
Antibacterial coatings have been accepted as a potent solution to the problem of medical-device-associated infections, which affect millions of patients worldwide. In this work, we present silver-nanoparticle-based antibacterial coatings that are highly potent against medically relevant bacteria such as $S$. epidermidis and $S$. aureus but do not have apparent cytotoxicity to primary human fibroblast cells. The silver nanoparticles used in the coatings are synthesized in the presence of trisodium citrate. At a given moment, 2-mercaptosuccinc acid is added to allow for reduction of nanoparticle size and to provide the well-defined surface architecture necessary for controllable surface immobilization. Important for application, the technology is facilitated by a functional-plasma-deposited polymer interlayer. This makes it applicable to the surface of medical devices manufactured from any type of material.
\end{abstract}

KEY WORDS: Silver nanoparticles, citrate reduction, antibacterial coating, plasma polymerization

\section{INTRODUCTION}

Lifesaving treatments and equipment such as heart valves or catheters can sometimes endanger patients' lives if they become the host of bacterial colonization and subsequently infection. ${ }^{1,2}$ These complex infections, which are acquired primarily during hospitalization [termed hospital acquired infections (HAIs)], are extremely difficult to treat and eradicate. ${ }^{3} \mathrm{~A}$ feasible prevention approach that has generated considerable attention over the last two decades consists of placing an appropriate antibacterial coating on the surfaces of medical devices. ${ }^{4,5}$ Coatings based on silver nanoparticles (AgNPs) have attracted attention because of their broad spectrum of antibacterial activity and unique physiochemical properties. ${ }^{6}$ Silver nanoparticles are applied extensively as antibacterial materials on devices because of their strong bactericidal action and capacity to disrupt biofilm formation. $^{7-9}$

Nanoparticles (NPs) are often purposely surface engineered with an appropriate capping agent to enhance their properties such as interaction with cells, immobilization 
to surfaces, targeted delivery, reduced inflammation, and cytotoxicity. ${ }^{10-15}$ Factors such as size, shape, aggregation state, capping agent and surface chemistry, dissolution, and solution properties (i.e., $\mathrm{pH}$ and ionic strength) can all influence the toxicity and antibacterial capability of AgNPs. ${ }^{6,16}$ Size and shape, in particular, have dominant control over many of the physiochemical characteristics of nanoscale materials including electromagnetic, optical, and catalytic activity as well as conductivity. ${ }^{17,18}$ Bacteria/nano-silver interactions are also dictated by the size and shape of silver nanoparticles. ${ }^{19-24}$ These facts have encouraged extensive research efforts on elaborating the synthetic routes to allow greater control of nanoparticle shape and size. ${ }^{19-24}$

Silver nanoparticles are commonly synthesized via chemical reduction methods. Typically, a metal salt precursor is reduced to its metallic form in the presence of an appropriate reducing agent. A capping agent is also added to assist with the control of nanoparticle growth, size, and colloidal stability. ${ }^{25}$ Among the variety of synthetic methods proposed for generation of silver nanoparticles, citrate reduction of silver nitrate is a popular synthetic procedure, which was first reported by Lee and Meisel in 1982..6 Citrate plays a dual role as a reductant and a stabilizer. The citrate reduction method typically results in silver crystallites of various shapes and size distributions in the range of 60-200 nm because trisodium citrate (TSC) is a weak reducing agent. ${ }^{17}$ The reaction stoichiometry can be expressed as follows:

$$
4 \mathrm{Ag}^{+}+\mathrm{C}_{6} \mathrm{H}_{5} \mathrm{O}_{7} \mathrm{Na}_{3}+2 \mathrm{H}_{2} \mathrm{O} \rightarrow 4 \mathrm{Ag}^{0}+\mathrm{C}_{6} \mathrm{H}_{5} \mathrm{O}_{7} \mathrm{H}_{3}+3 \mathrm{Na}^{+}+\mathrm{H}^{+}+\mathrm{O}_{2} \uparrow 27
$$

The nanoparticle size and shape is affected by the ratio of [sodium citrate] $\left[\mathrm{Ag}^{+}\right] .^{28}$ A drawback of the citrate reduction method is the nanoparticle surface structure. The particles are capped by physisorbed citrate ions and products of the reduction, which provide stabilization of the colloidal suspension. However, the surface structure is not well defined. These ions may separate from the particle surface upon changing $\mathrm{pH}$ and/or ionic strength as has been demonstrated with gold nanoparticles by Zhu et al. ${ }^{29}$ Furthermore, this undefined surface structure may make attachment of nanoparticles to solid surfaces challenging and irreproducible. For generation of antibacterial surfaces and other applications, controlled immobilization of nanoparticles to surfaces is essential.

In this work, we present a method to control the size of silver nanoparticles and their surface structure by introducing a co-capping agent during the nanoparticle growth phase. We selected 2-mercaptosuccinic acid (MSA) as the co-capping agent for several reasons. The MSA molecule contains a thiol group, which is well known for its affinity to silver and other metals. ${ }^{30}$ We hypothesized that, upon introduction, MSA binds to the growing nanoparticles through its thiol group forming a self-assembled monolayer (SAM) on the surface of the nanoparticle, thus terminating their growth. Second, the MSA molecule carries two carboxyl acid groups. Upon binding to the nanoparticle surface, the two carboxyl acid groups point outward. If the $\mathrm{pH}$ is $\geq 5$, a negative charge occurs on the surface of the nanoparticles that stabilizes the colloidal solution. In addition, this negative charge facilitates immobilization to solid surfaces, carrying a positive 
charge such as those modified with a plasma polymer coating deposited from the vapor of allylamine. A plasma-deposited interlayer was selected with the purpose of applying the antibacterial coating to any type of substrate material because the technique is widely accepted to be substrate independent. ${ }^{31,32}$ Third, we have recently shown that antibacterial coatings based on mercaptosuccinic acid capped silver nanoparticles have excellent efficacy and biocompatibility. ${ }^{33}$

\section{MATERIALS AND METHODS}

\section{A. Material}

\section{Thin film preparation}

Allyamine (AA) (98\%), silver nitrate (AgNO 99.99\%), and trisodium citrate (TSC, C H O Na ), 2-mercaptosuccinic acid (97\%) of analytical grade purity were supplied by A $^{5}$ drich $^{3}$ (Australia) and used as starting materials without further purification. Hydrochloric acid (36\%) was supplied by Ajax Finechem Pty. Ltd (Australia) and nitric acid $\left(\mathrm{HNO}_{3}, 70 \%\right)$ was purchased from Sigma-Aldrich. All solution preparation and glassware cleaning procedures were performed using ultrapure (Milli-Q) water (resistivity $18.2 \Omega$ ). All glassware and magnet stirrers were soaked in aqua regia solution $(3: 1$ conc. $\mathrm{HCl}$ : conc. $\mathrm{HNO}_{3}$ ) and were then rinsed thoroughly with the Milli-Q water before performing the nanoparticle solution preparation.

\section{Antibacterial study}

Staphylococcus epidermidis strain ATCC 35984, Staphylococcus aureus strain ATCC 4330 [methicillin-resistant Staphylococcus aureus (MRSA)], were cultured in tryptone soya broth (TSB, Oxoid, UK) at $37^{\circ} \mathrm{C}$ and $60 \%$ humidity. Safranin-O and acetic acid glacial were purchased from Chemical Supply (Australia).

\section{Cell viability study}

Human dermal fibroblasts (HDFs) were harvested and grown as described previous$1 \mathrm{y}^{34}$ from split-thickness skin grafts (STSGs) obtained from scavenged tissue specimens following routine breast reductions and abdominoplasties. All patients gave informed consent for their skin to be used for research through a protocol approved by the Ethics Committee at the Queen Elisabeth Hospital and the University of South Australia Human Ethics Committee. Briefly, fibroblasts were grown in fibroblast culture medium (FCM) consisting of Dulbecco's modified Eagle medium (DMEM) high glucose (Glutamax, Gibco, Life Technologies, Australia), 10\% v/v fetal calf serum (FCS, Ausgenex, 
Australia), $0.625 \mu \mathrm{g} / \mathrm{mL}$ amphotericin B (Sigma-Aldrich, Australia), $100 \mathrm{IU} / \mathrm{mL}$ penicillin and $100 \mathrm{mg} / \mathrm{mL}$ streptomycin (Gibco, Life Technologies, Australia) in an incubator at $37^{\circ} \mathrm{C}$, under a $5 \% \mathrm{CO}_{2}$ humidified atmosphere. The medium was changed every 3-4 days until the cells were $80 \%$ confluent. HDFs collected between passages 3 and 9 were used in the study. Cells were stained with the Phalloatoxin "Alexa Fluor 488® phalloidin" (a filamentous actin probe) and DAPI dilactate purchased from Life Technologies, Invitrogen.

\section{B. Instrumental}

UV-visible spectra were recorded on a Cary 5 UV-vis spectrometer (Varian Australia Pty, Ltd.) from $250 \mathrm{~nm}$ to $700 \mathrm{~nm}$. Milli-Q water was used as the blank, and $1 \mathrm{ml}$ of sample was diluted in ratio 3:1 with Milli-Q before UV-vis analysis. Dynamic light scattering (DLS) was used to measure the average size and the size distribution using a PSS Nicomp Particle Sizer 380 (Nicomp Particle Sizing Systems, USA). To study the morphology of polymer particles, samples were sputter coated with $5 \mathrm{~nm}$ of $\mathrm{Cr}$ using a Quorum Q150T sputter-coating system. Subsequent imaging of AgNP films was conducted using an FEI Quanta 450 FEG ESEM operated in high vacuum mode at an electron-beam-accelerating voltage of $30 \mathrm{kV}$. Samples were imaged using both the secondary and backscattered electron signals. Particle sizing analysis was performed with the NIH Image $\mathrm{J}$ software. Transmission electron microscope (TEM) images were acquired using a Philips CM200 TEM. The zeta potential of nanoparticles was measured in a $10^{-3} \mathrm{M}$ potassium chloride $(\mathrm{KCl})$ solution $(100 \mu \mathrm{l}$ of sample in $900 \mu \mathrm{l}$ $\mathrm{KCl}$ ) with a Zeta Nanosizer (Malvern Instruments, UK) at $25^{\circ} \mathrm{C}$. X-ray photoelectron spectroscopy (XPS) spectra were recorded on a SPECS SAGE spectrometer. Processing and component fitting of high-resolution spectra were performed with CasaXPS software.

\section{Fabrication of Antibacterial Coating}

The preparation of the thin film consisting of silver nanoparticles in an amine-rich film was carried out in three steps: (1) synthesis of silver nanoparticles coated with tri-sodium citrate and 2-mercaptosuccinic acid (AgNPs@TSC-MSA), (2) fabrication of the allylamine plasma polymer thin film (AApp), and (3) immobilization of nanoparticles on the surface (AApp+AgNPs@TSC-MSA).

\section{Synthesis of silver nanoparticles}

The AgNPs@TSC-MSA nanoparticles were prepared using a slightly modified version of the Lee and Meisel protocol. ${ }^{26}$ A total of $1 \mathrm{~mL}$ of $1 \mathrm{wt} \% \mathrm{TSC}$ was added to $50 \mathrm{~mL}$ 
of boiling $1 \mathrm{mM}$ silver nitrate solution, and heating was continued until the solution turned light yellow in color, indicating the formation of nanoparticles. The change in color from colorless to yellow is due to the reduction and agglomeration of the $\mathrm{Ag}^{+}$ ions to metallic silver. Heating was continued for an additional $15 \mathrm{~min}$. The suspension was then allowed to cool down to room temperature under vigorous stirring; $5 \mathrm{ml}$ of $1 \%$ MSA was added during this cooling period. The final color was golden brown. The same procedure was followed to prepare the AgNPs@TSC nanoparticles; however, after cooling the suspension to room temperature, there was no additional step. At this step, the nanoparticles are coated with TSC.

\section{Fabrication of allylamine plasma polymer film}

Plasma polymerization was carried out in a custom-built reactor described elsewhere ${ }^{35}$ using a $13.56 \mathrm{MHz}$ plasma generator (Advanced Energy, USA) and a matching network (Advanced Energy, USA). The 13-mm Thermanox coverslip substrates (Thermo Fisher Scientific, USA) were cleaned with ethanol and Milli-Q water before being placed in the reactor chamber. Then they were cleaned by oxygen plasma for $2 \mathrm{~min}$ at pressure of $2.5 \times 10^{-2}$ mbar using a power of $20 \mathrm{~W}$. The deposition of the allylamine (AA) plasma polymer was carried out at a monomer pressure of $2 \times 10^{-3}$ mbar. The input radiofrequency (RF) power was $10 \mathrm{~W}$; the monomer flow rate was $10 \mathrm{sccm}$; and the time of deposition was $5 \mathrm{~min}$. These conditions result in nitrogen-rich films with a thickness of $24 \mathrm{~nm}$. Allylamine plasma polymer (AApp) substrates are kept sealed overnight at ambient temperature before undergoing any further modification, to let the polymer surface to find its stable structure with minimum interfacial energy. The allylamine was plasma polymerized on silicon wafers for SEM analysis, and on glass and Thermanox coverslips for bacterial and cell culture studies.

\section{Immobilization of nanoparticles on the surface}

AApp-coated substrates were immersed in the solution of AgNPs@TSC-MSA for 24 h at ambient temperature $\left(25^{\circ} \mathrm{C}\right)$. After immersion, the samples were rinsed thoroughly with Milli-Q water and dried with nitrogen and were kept sealed.

\section{Cell Viability and Morphology Study}

\section{Cell viability}

Samples were placed in 24-well tissue culture plates. Human dermal fibroblasts were seeded at a density of $1 \times 10^{4}$ cells per well in fibroblast culture media. After 2 days, cell viability was measured by the resazurin assay. A stock solution of $110 \mu \mathrm{g} / \mathrm{ml}$ resazurin 
sodium salt (Sigma-Aldrich, Australia) was prepared in PBS and filter sterilized using a $0.02-\mu \mathrm{m}$ filter. This solution was then diluted 1:10 in fibroblast culture medium. A total of $500 \mu \mathrm{L}$ of the resazurin solution was added to each sample. After 4 hours, $200 \mu \mathrm{L}$ of the reduced solution was removed from each well and placed into a well of a 96-well plate. Then the florescent intensity was read using a plate reader $(\lambda \mathrm{ex}=544 \mathrm{~nm}$ and $\lambda \mathrm{em}$ $=590 \mathrm{~nm}$ ).

\section{Cell morphology}

Finally, the samples were fixed in 4\% paraformaldehyde in PBS for immunocytochemistry staining of the cytoskeleton to assess cell morphology. Fixed cells were incubated at room temperature with $0.1 \%(\mathrm{v} / \mathrm{v})$ Triton X-100 for 5 minutes to permeablize the membranes. Phallotoxin Alexa -488 and DAPI to final concentrations of $0.165 \mu \mathrm{M}$ and $0.1 \mu \mathrm{g} / \mathrm{mL}$, respectively, in $200 \mu \mathrm{L}$ of PBS was added to each sample, and the samples were incubated further in the dark. Excess dye was washed from the samples, and the samples were imaged using a Nikon A1-R confocal laser scanning microscope using NIS elements AR software.

\section{E. Surface-Immobilized Antibacterial Activity}

Bacteria were inoculated onto blood agar plates and incubated overnight at $37^{\circ} \mathrm{C}$. Individual bacterial colonies were isolated and incubated overnight at $37^{\circ} \mathrm{C}$ in $10 \mathrm{~mL}$ Tryptic Soya Broth (TSB). A total of $1 \mathrm{~mL}$ of solution was diluted in $9 \mathrm{ml}$ of fresh TSB and incubated for $2 \mathrm{~h}$ at $37^{\circ} \mathrm{C}$. Approximately $1 \times 10^{6} \mathrm{cfu} / \mathrm{ml}$ of bacteria was prepared using fresh TSB. Immobilized AgNPs@MSA on AApp surfaces were placed in 24-well plates. Each well was filled with $400 \mu 1$ of diluted culture of bacteria $(1 \times$ $10^{6} \mathrm{cfu} / \mathrm{ml}$ ) in TSB. Two sets of AApp cover slips covered with $400 \mu \mathrm{l}$ TSB were used as positive and negative control. The sample box was incubated in a container with moist foam for $4 \mathrm{~h}$ at $37^{\circ} \mathrm{C}$; the broth in each well was replaced with $400 \mu \mathrm{l}$ fresh TSB and incubated in similar fashion for $24 \mathrm{~h}$ at $37^{\circ} \mathrm{C}$. After the second incubation period, the TSB was removed, and wells were rinsed twice with $400 \mu$ of sterile saline $(0.9 \%$ sterile solution of sodium chloride in water) to clean any loose biofilm formed on the surface. The biofilm formed on the other side of each coverslip was removed with a swab immersed in ethanol (EtOH, 70\%). A total of $400 \mu$ of Safranin-O (0.1\%, Ajax Chemical) solution was added to each well to stain the biofilm adhered to the surface. After $30 \mathrm{~min}$, the Safranin-O solution was removed, and the wells were rinsed twice with $400 \mu \mathrm{L}$ of Milli-Q water to remove excess Safranin-O and allowed to dry at ambient temperature. $400 \mu \mathrm{L}$ of acetic acid (AcOH, 33\%) was introduced into each well to lyse the bacterial cells and release the trapped dye. A total of $100 \mu \mathrm{L}$ from each well was transferred into a 96-well plate, and the optical density (OD) was measured by an ELISA plate reader at $490 \mathrm{~nm}$. 


\section{F. Statistical Analysis Methods}

All statistical analyses were performed on GraphPad Prism 5, and a one-way ANOVA test was performed on data. The Dunnett's posttest was performed on antibacterial and fibroblast cell results. In antibacterial and fibroblast cell studies, AApp and glass surfaces were chosen, respectively, as controls against which data were compared.

\section{RESULTS AND DISCUSSION}

Table 1 summarizes the physical characteristic of the solution prepared using MSA as the co-capping agent and the solution using TSC only.

Both synthetic procedures resulted in clear, golden-brown solutions that had good colloidal stability of $>6$ months. The colloids were negatively charged as a result of presence of carboxyl groups. The zeta potential of the MSA stabilized AgNPs was approximately $-40 \mathrm{mV}$ versus $-22.5 \mathrm{mV}$ for those synthesized in the presence of TSC only. Notably, even at lower $\mathrm{pH}$, the MSA-modified nanoparticles had higher negative surface potential, which could be attributed to the well-arranged self-assembled monolayer on the surface of the nanoparticles having outward- pointing $\mathrm{COOH}$ groups. This stronger negative surface potential at lower $\mathrm{pH}$ is beneficial for immobilization of the nanoparticles to positively charged amine rich plasma polymer coatings, which have a higher extent of protonation when the solution $\mathrm{pH}$ is reduced. ${ }^{36}$ Dynamic light scattering measurements showed dual size distributions for both types of nanoparticles. For the AgNPs@TSC-MSA, the predominant sizes were 5 and 34 nm, while for the AgNPs@ TSC, the sizes were 5 and $58 \mathrm{~nm}$.

TABLE 1: Characteristic of colloidal solutions of AgNPs capped with MSA and those synthesizes using TSC only.

\begin{tabular}{lll}
\hline Characteristics & AgNPs@TSC-MSA & AgNPs@TSC \\
\hline Color & Golden brown clear solution & $\begin{array}{l}\text { Golden brown clear solu- } \\
\text { tion }\end{array}$ \\
Stability & $6+$ months & $6+$ months \\
pH & $4 \pm 0.5$ & $6 \pm 0.2$ \\
Zeta potential (mV) & -40 & -22.5 \\
$\begin{array}{l}\text { NP-sized Malvern DLS (nm) } \\
\begin{array}{l}\text { Max extinction in the UV-visible } \\
\text { spectra (nm) }\end{array}\end{array}$ & $5(21.8 \%), 34(78.2 \%)$ & $5(17.0 \%), 58(83.0 \%)$ \\
\hline
\end{tabular}



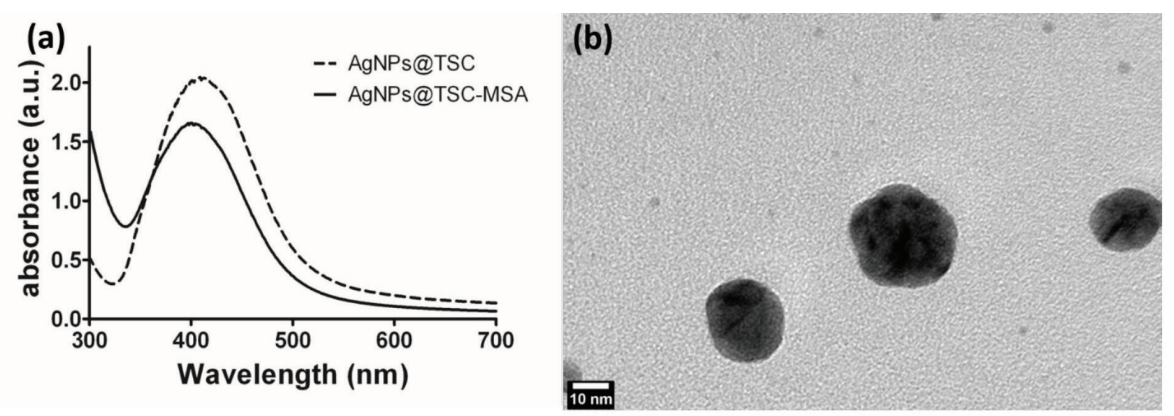

FIG. 1: (a) UV-Visible spectra of colloidal solution of AgNPs@TSC-MSA (maximum absorbance at $405 \mathrm{~nm}$ ) and AgNPs@TSC (maximum absorbance at $420 \mathrm{~nm}$ ); (b) TEM micrograph of AgNPs@TSC-MSA. (Scale bar represents 10 nm)

Figure 1a shows representative UV-visible spectra of the AgNPs@TSC-MSA and AgNPs@TSC colloidal suspensions. The UV-visible spectrum of the colloidal solution was measured at $25^{\circ} \mathrm{C}$. Recorded spectra were analyzed for peak position, peak width, and area under the peak of the plasmon resonance. The spectra were fitted to determine the position of the plasmon peak and excluded the wavelength range of $350-650 \mathrm{~nm}$ to avoid fit distortions. The characteristic plasmon resonance bands of AgNPs is governed by parameters such as the size, shape, morphology, composition and dielectric environment of the prepared nanoparticles. ${ }^{37}$ Silver nanoparticles generated by citrate reduction are reported to give a plasmon resonance peak at $\sim 420-430$ $\mathrm{nm}^{17,38}$ (Fig. 1a). This is consistent in our synthesis, i.e., the peak is at $420 \mathrm{~nm}, \mathrm{FWHM}$ 108.17, and 2.044 a.u. absorbance intensity. The colloidal solution of AgNPs@TSCMSA gave the absorption maxima at $\sim 05 \mathrm{~nm}, 1.744$ a.u. absorbance intensity, and FWHM 128.03 (Fig. 1a). This absorption maxima suggests the existence of particles, with shapes between spherical and dodecahedral ${ }^{39}$ and a moderate dispersity in particle sizes. This assumption is confirmed by TEM images of nanoparticles, which, in the case of AgNPs@TSC-MSA, have a semi-spherical AgNPs shape and size of $25 \pm 10$ nm and $8 \pm 4$ nm (Fig. 1b).

For the purpose of applying an antibacterial coating, the nanoparticles need to be immobilized to solid surfaces. A 20-nm thin plasma polymer film deposited from vapor of allylamine was placed on silicon wafers and Thermanox coverslips. These films are known to carry a population of amine groups which protonate when placed in solution of $\mathrm{pH}$ below $8 .{ }^{36}$ As shown in Table 1, both synthetic procedures lead to silver nanoparticles carrying a negative surface charge which makes them suitable for immobilization to positively charged surfaces via electrostatic coupling. The film was characterized by SEM and XPS to study surface morphology and chemistry, respectively.

Figure 2 shows SEM images of the AgNPs@TSC (left) and AgNPs@TSC-MSA (right) after immobilization on AApp modified silicon wafers. Higher magnification images were also added as inserts. Nanoparticles immobilized on AApp modified Ther- 
manox coverslips (used for the biological studies outlined below) had brownish color indicating the presence of attached silver nanoparticles. Images of Thermanox coverslips after immobilization of silver nanoparticles are shown as inserts in Figure 2. The SEM images show that both nanoparticle types readily attach to the plasma polymer modified surface. Clearly, the AgNPs@TSC are significantly larger, most having diameters of $\sim 50-60 \mathrm{~nm}$. The results of the UV-Vis, DLS, TEM, and SEM analysis clearly demonstrate that adding MSA results in smaller nanoparticles, which indicates that using a co-capping agent successfully inhibited bacterial growth. Because smaller nanoparticles are known to have more potent antibacterial efficacy, ${ }^{19}$ all biological studies were carried out with AgNPs@TSC-MSA.

The XPS survey spectra of the samples obtained after 24 hours of immobilization

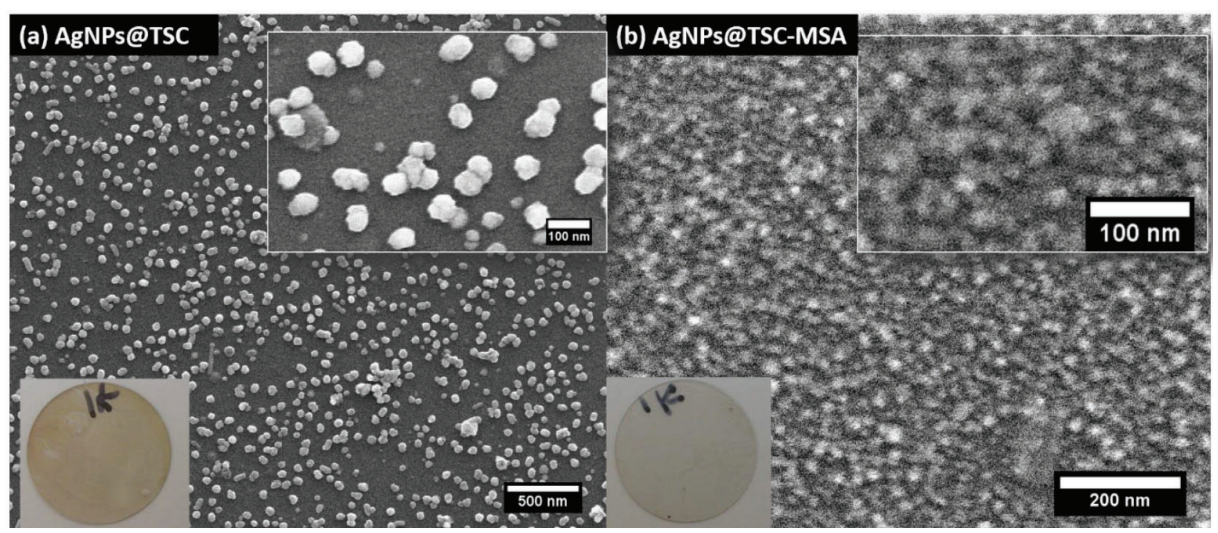

FIG. 2: SEM micrograph of (a) AgNPs@TSC and (b) AgNPs@TSC-MSA immobilized onto AApp modified silicon wafer. The scale bars are $500 \mathrm{~nm}$ and $200 \mathrm{~nm}$ on the lower magnification images (a) and (b), respectively, and $100 \mathrm{~nm}$ on the inserts in the top right corner). The insert in the left bottom corner of (a) and (b) shows coated Thermanox coverslips after immobilization of silver nanoparticles

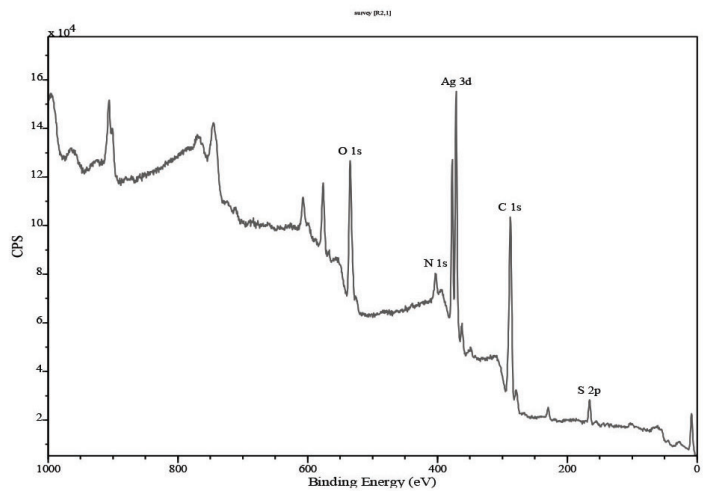

FIG. 3: XPS survey spectrum of immobilized AgNPs@TSC-MSA on AApp film 
shows the presence of five dominant chemical elements: silver, carbon, nitrogen, oxygen, and sulfur (Figures 2 and 3). The presence of sulfur is indicative of the layer of MSA capping the silver nanoparticles.

The influence of the immersion duration on the concentration of immobilized silver nanoparticles as determined from XPS analysis is shown in Figure 4.

Two medically significant pathogenic bacterial species, Staphylococcus epidermidis (S. epidermidis) and Staphylococcus aureus (S. aureus), were chosen to evaluate the antibacterial efficacy of coatings produced using AgNP@TSC-MSA. These pathogens were selected because they are significant contributors to the increasing problem of biomedical-device-associated infections. ${ }^{40,41}$ The presence of bacteria on the surface and biofilm development was assessed by of Safranin-O staining. To evaluate the effect of nanoparticles surface concentration of bacterial colonization, we examined coatings that were produced after immobilization for 1,6 , and $24 \mathrm{~h}$.

Figure 5 shows that the coatings prepared by overnight immersion in the colloidal solution had superior activity, while 6-h samples(containing $\sim 4.5 \%$ silver as determined by XPS) were only partially affect bacterial attachments, growth, and biofilm formation. The samples produced at $1 \mathrm{~h}$ immersion time (containing $\sim 1 \%$ silver as determined by XPS) had no inhibitory activity against $S$. epidermidis.

These results demonstrate that the antibacterial capability of the surface is highly dependent on the surface concentration of AgNPs. Silver nanoparticles affected the two bacteria differently. A higher concentration of surface immobilized silver nanoparticles was required to stop the growth of $S$. epidermidis. This is not surprising because this microorganism is known to have a highly surface adherent and to be a very strong biofilm former. ${ }^{40}$ Nevertheless, samples obtained after $24 \mathrm{~h}$ of immobilization show a reduction in the biofilm level of $85 \%$ relative to the control. A lesser concentration of silver nanoparticles was required to inhibit the surface growth of $S$. aureus. A 1 At $\%$ quantity of silver nanoparticles on the surface was sufficient to reduce the number of bacteria by

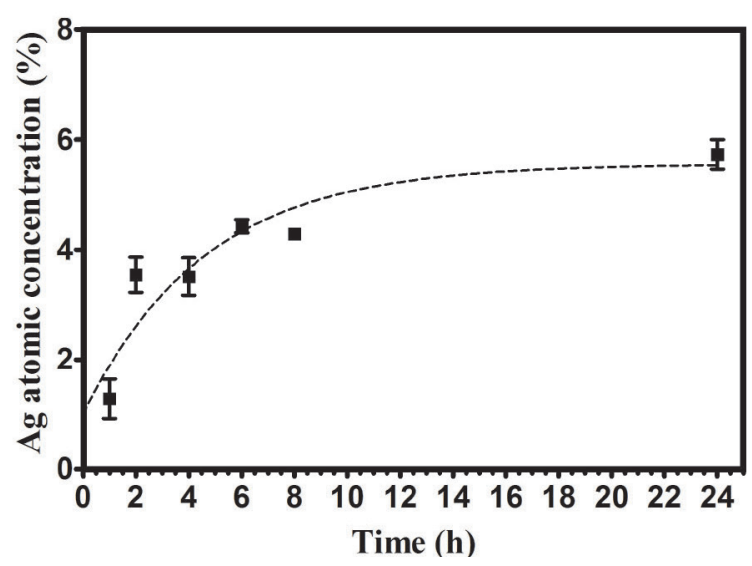

FIG. 4: Effect of immersion duration on the final concentration of silver nanoparticles immobilized on AApp thin film determined by XPS 

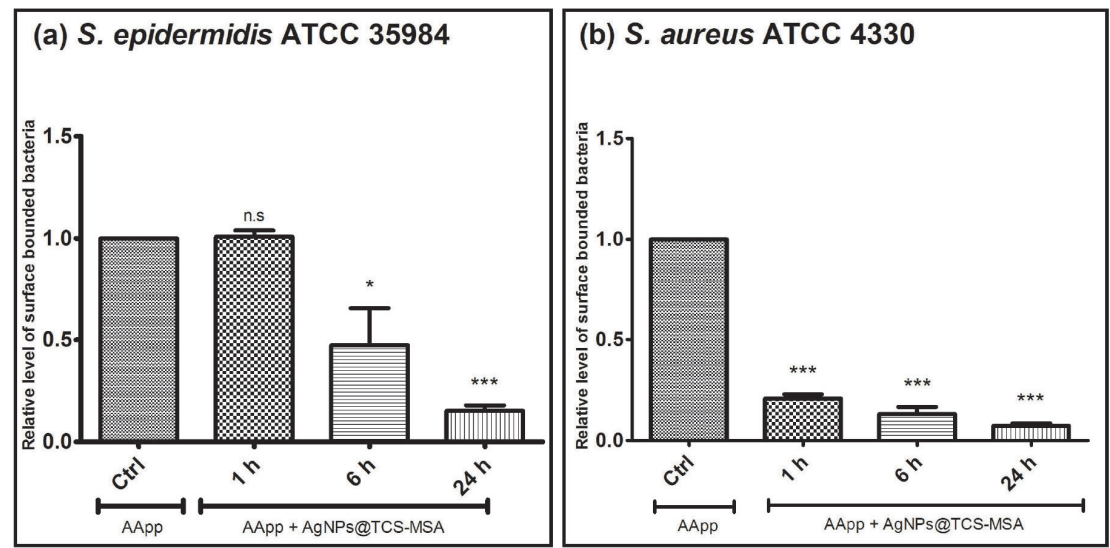

FIG. 5: Levels of inhibition of surface colonization of (a) S. epidermidis ATCC 35984 and (b) S. aureus ATCC 4330 on AApp-coated substrate as a control and AgNPs@TSC-MSA immobilized for 1, 6, 24 h. $n=3$. Data are expressed as mean \pm SEM. Symbol definition compared with control: n.s., not significant; ${ }^{*} \mathrm{p}<0.05, * * * \mathrm{p}<0.001$

(a) Fibroblast viability from resazurin assay

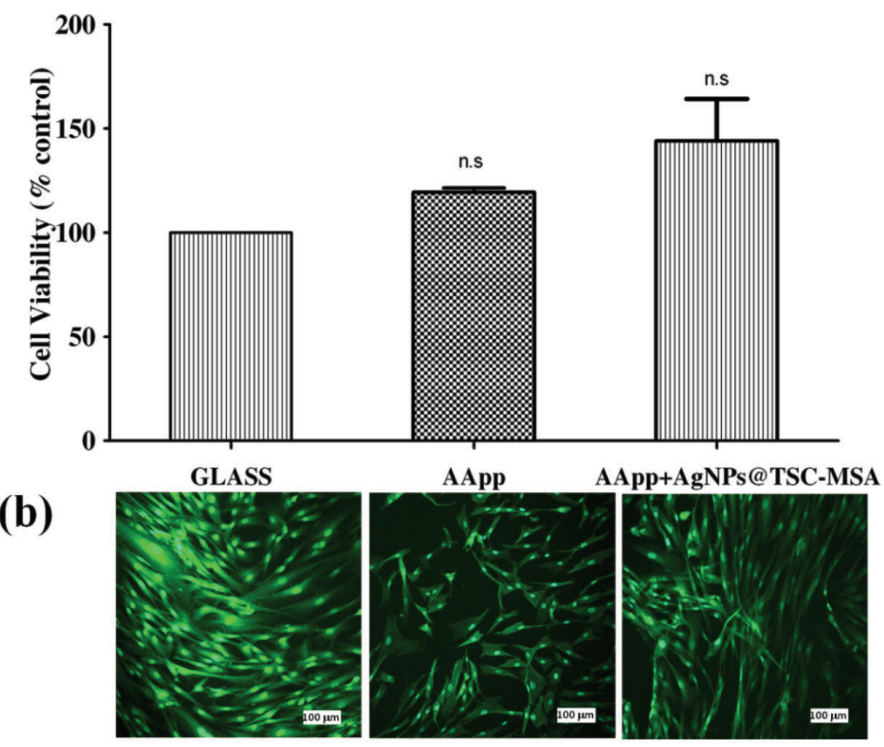

FIG. 6: (a) Relative fibroblast viability determined from Resazurin assay compared to a glass only control. $n=3$. Data are expressed as mean \pm SEM. Symbol definition compared with control: n.s., statistically not significant; (b) confocal microscopy images of fibroblast cells cultured on glass (left), AApp (middle), and AgNPs@TSC-MSA + AApp (right) substrates. (Scale = $100 \mu \mathrm{m}$ ) 
$80 \%$ relative to the control. This is expected because these species typically target tissue and do not form a strong biofilm. ${ }^{42,43}$

Finally, we investigated the potential toxicity of the antibacterial coatings generated this work to primary human dermal fibroblast cells via Resazurin assay. Surfaces were coated with the highest density of silver nanoparticles achieved after $24 \mathrm{~h}$ immersion (6 At\% of silver as determined by XPS). The effect of plasma polymer film on the cells was also investigated by culturing the cells on the coverslips coated only with the AApp thin film. The data was then compared with cells grown on uncoated glass coverslips (Figure 6a). AApp coatings have previously been shown to be non-cytotoxic and to enhance fibroblast growth and attachment. ${ }^{44}$ Cells cultured on substrates coated with AApp thin film are more metabolically active compared to the glass control. The metabolic activity of the cell cultured on the silver-nanoparticle-modified surfaces appears even higher; however, this was not statistically significant $(p=0.0953)$. Laser scanning confocal microscopy images of the cells grown on the three types of surfaces after 24 $\mathrm{h}$ of incubation are shown in Figure $6 \mathrm{~b}$. Our collective results suggest that the coatings prepared by immobilization of surface-modified silver nanoparticles at its bactericidal concentration are not cytotoxic to eukaryotic cells. These results are in accordance with our expectations and with previously published studies that have indicated that mammalian cells may be able to tolerate higher amounts of silver than bacteria. ${ }^{45}$ It should be stressed that, unlike the usual research practice, the data in our study were obtained from a culture of primary cells and did not utilize immortalized cell lines.

Surface modifications of biomedical devices, such as implants, wound dressings, and catheters, have attracted much attention as a tool to reduce the incidence of device-related infections. Coatings with the capacity to release antimicrobial agents have been proposed as a potent solution. ${ }^{4,46}$ However, the side effects of such a strategy, including toxicity, should be considered. ${ }^{47}$ The silver-nanoparticle-based approach presented in this paper leads to an effective antibacterial coating with no induced toxicity toward primary human fibroblast cells. We are able to control the amount of AgNPs attached to the surface by controlling the time of immobilization. The nanoparticles are prepared in a controllable manner by introducing MSA during the growth phase, which results in the generation of particles with a relatively narrow range of size and shape distribution. This is important because it allows more predictable assessment of antibacterial properties and potential cytotoxicity. The surface-independent procedure for the immobilization of silver nanoparticles on a functional-plasma-deposited polymer interlayer has possible applications for antibacterial coatings that can be applied to wound dressings and implantable biomedical devices.

\section{CONCLUSION}

Herein, we have reported the fabrication of a silver-nanoparticle-based antibacterial coating with no apparent toxicity to primary fibroblasts. The addition of MSA as a co-capping agent enables control of size, shape, and dispersion of silver nanoparticles. 
The immobilization of silver nanoparticles to solid surfaces was facilitated by a functional-plasma-polymer-deposited interlayer. Control over nanoparticles surface concentration was achieved by modifying the time of surface immobilization. The antibacterial properties of the coatings were assessed in cultures of two bacterial species of medical significance, S. epidermidis and $S$. aureus. The levels of bacterial colonization and biofilm formation were reduced by $>85 \%$ in the case of $S$. epidermidis and by $>95 \%$ in the case of $S$. aureus. The potential cytotoxicity of the coatings was assessed in cultures of primary human fibroblasts. Even the highest silver-nanoparticle surface concentration did not seem to have any cytotoxic effect, as the cells had viability even higher than on the control surface, adhered in great numbers, and showed healthy morphology. The high antibacterial efficacy and absence of cytotoxicity make the coatings developed in this study excellent candidates for application on implantable medical device surfaces as well as catheters and wound dressings.

\section{ACKNOWLEDGMENTS}

KV thanks ARC for support through fellowship FT100100292. The authors would also like to acknowledge Professor Michael Roberts from the Division of Health Sciences, School of Pharmacy and Medical Sciences, University of South Australia for the provision of skin from which the HDFs were isolated.

\section{REFERENCES}

1. Weston D. Types of healthcare associated infection. Infection prevention and control. John Wiley \& Sons Ltd; 2008. p. 85-109.

2. Antoci V, Adams CS, Parvizi J, Davidson HM, Composto RJ, Freeman TA, Wickstrom E, Ducheyne P, Jungkind D, Shapiro IM. The inhibition of Staphylococcus epidermidis biofilm formation by vancomycin-modified titanium alloy and implications for the treatment of periprosthetic infection. Biomaterials. 2008;29(35):4684-90.

3. Paitoonpong L, Wong CKB, Perl TM. Healthcare-associated infections. Infect Dis Epidemiol. 2013. p. $369-466$.

4. Montali A. Antibacterial coating systems. Injury. 2006;37(2, Supplement):S81-S6.

5. Fortunati E, Mattioli S, Visai L, Imbriani M, Fierro JLG, Kenny JM, Armentano I. Combined effects of Ag nanoparticles and oxygen plasma treatment on PLGA morphological, chemical, and antibacterial properties. Biomacromolecules. 2013;14(3):626-36.

6. Marambio-Jones C, Hoek EV. A review of the antibacterial effects of silver nanomaterials and potential implications for human health and the environment. J Nanoparticle Res. 2010;12(5):1531-51.

7. Kalishwaralal K, BarathManiKanth S, Pandian SRK, Deepak V, Gurunathan S. Silver nanoparticles impede the biofilm formation by Pseudomonas aeruginosa and Staphylococcus epidermidis. Colloids and Surfaces B: Biointerfaces. 2010;79(2):340-4.

8. Rai MK, Deshmukh SD, Ingle AP, Gade AK. Silver nanoparticles: the powerful nanoweapon against multidrug-resistant bacteria. J Appl Microbiol. 2012;112(5):841-52.

9. Furno F, Morley KS, Wong B, Sharp BL, Arnold PL, Howdle SM, Bayston R, Brown PD, Winship PD, Reid HJ. Silver nanoparticles and polymeric medical devices: a new approach to prevention of infection? J Antimicrobial Chemother. 2004;54(6):1019-24. 
10. Hühn D, Kantner K, Geidel C, Brandholt S, De Cock I, Soenen SJH, Rivera_Gil P, Montenegro J-M, Braeckmans K, Müllen K, Nienhaus GU, Klapper M, Parak WJ. Polymer-coated nanoparticles interacting with proteins and cells: focusing on the sign of the net charge. ACS Nano. 2013;7(4):3253-63.

11. Malynych S, Luzinov I, Chumanov G. Poly(vinyl pyridine) as a universal surface modifier for immobilization of nanoparticles. J Phys Chem B. 2002;106(6):1280-5.

12. Wang Y, Newell BB, Irudayaraj J. Folic acid protected silver nanocarriers for targeted drug delivery. J Biomed Nanotechnol. 2012;8(5):751-9.

13. Mishra B, Patel BB, Tiwari S. Colloidal nanocarriers: a review on formulation technology, types and applications toward targeted drug delivery. Nanomed Nanotechnol Biol Med. 2010;6(1):9-24.

14. Jena P, Mohanty S, Mallick R, Jacob B, Sonawane A. Toxicity and antibacterial assessment of chitosancoated silver nanoparticles on human pathogens and macrophage cells. Int J Nanomed. 2012;7:1805.

15. Lewinski N, Colvin V, Drezek R. Cytotoxicity of nanoparticles. Small. 2008;4(1):26-49.

16. Fabrega J, Luoma SN, Tyler CR, Galloway TS, Lead JR. Silver nanoparticles: behaviour and effects in the aquatic environment. Envir Int. 2011;37(2):517-31.

17. Pillai ZS, Kamat PV. What factors control the size and shape of silver nanoparticles in the citrate ion reduction method? J Phys Chem B. 2004;108(3):945-51.

18. Burda C, Chen X, Narayanan R, El-Sayed MA. Chemistry and properties of nanocrystals of different shapes. Chem Revs. 2005;105(4):1025-1102.

19. Martinez-Castanon G, Nino-Martinez N, Martinez-Gutierrez F, Martinez-Mendoza J, Ruiz F. Synthesis and antibacterial activity of silver nanoparticles with different sizes. J Nanoparticle Res. 2008;10(8):1343-8.

20. Agnihotri S, Mukherji S, Mukherji S. Size-controlled silver nanoparticles synthesized over the range 5-100 nm using the same protocol and their antibacterial efficacy. RSC Advances. 2014;4(8):3974-83.

21. Liu H-L, Dai SA, Fu K-Y, Hsu S-h. Antibacterial properties of silver nanoparticles in three different sizes and their nanocomposites with a new waterborne polyurethane. Int J Nanomed. 2010;5:1017.

22. Lu Z, Rong K, Li J, Yang H, Chen R. Size-dependent antibacterial activities of silver nanoparticles against oral anaerobic pathogenic bacteria. J Mater Sci: Mater Med. 2013;24(6):1465-71.

23. Pal S, Tak YK, Song JM. Does the antibacterial activity of silver nanoparticles depend on the shape of the nanoparticle? A study of the Gram-negative bacterium Escherichia coli. Appl Environ Microbiol. 2007;73(6):1712-20.

24. Morones JR, Elechiguerra JL, Camacho A, Holt K, Kouri JB, Ramírez JT, Yacaman MJ. The bactericidal effect of silver nanoparticles. Nanotechnology. 2005;16:2346.

25. García-Barrasa J, López-de-Luzuriaga J, Monge M. Silver nanoparticles: synthesis through chemical methods in solution and biomedical applications. Central Eur J Chem. 2011;9(1):7-19.

26. Lee PC, Meisel D. Adsorption and surface-enhanced Raman of dyes on silver and gold sols. J Phys Chem. 1982;86(17):3391-5.

27. Šileikaite A, Prosyčevas I, Puišo J, Juraitis A, Guobienė A. Analysis of silver nanoparticles produced by chemical reduction of silver salt solution. Mater Sci-Medzg. 2006;12:287-91.

28. Jiang XC, Chen CY, Chen WM, Yu AB. Role of citric acid in the formation of silver nanoplates through a synergistic reduction approach. Langmuir. 2009;26(6):4400-8.

29. Zhu T, Vasilev K, Kreiter M, Mittler S, Knoll W. Surface modification of citrate-reduced colloidal gold nanoparticles with 2-mercaptosuccinic acid. Langmuir. 2003;19(22):9518-25.

30. Love JC, Estroff LA, Kriebel JK, Nuzzo RG, Whitesides GM. Self-assembled monolayers of thiolates on metals as a form of nanotechnology. Chem Revs-Columbus. 2005;105(4):1103-70.

31. Vasilev K, Michelmore A, Griesser HJ, Short RD. Substrate influence on the initial growth phase of plasma-deposited polymer films. Chem Commun. 2009(24):3600-2.

32. Vasilev K, Michelmore A, Martinek P, Chan J, Sah V, Griesser HJ, Short RD. Early stages of growth of plasma polymer coatings deposited from nitrogen-and oxygen-containing monomers. Plasma Proc Polymer. 2010;7(9-10):824-35.

33. Taheri S, Cavallaro A, Christo SN, Smith LE, Majewski P, Barton M, Hayball JD, Vasilev K. Substrate 
independent silver nanoparticle based antibacterial coatings. Biomaterials. 2014;35(16):4601-9.

34. MacNeil S, Shepherd J, Smith L. Production of tissue-engineered skin and oral mucosa for clinical and experimental use. 3D Cell Culture: Springer; 2011. p. 129-53.

35. Whittle JD, Barton D, Alexander MR, Short RD. A method for the deposition of controllable chemical gradients. Chem Commun. 2003(14):1766-7.

36. Mierczynska A, Michelmore A, Tripathi A, Goreham RV, Sedev R, Vasilev K. pH-tunable gradients of wettability and surface potential. Soft Matter. 2012;8(32):8399-404.

37. Kelly KL, Coronado E, Zhao LL, Schatz GC. The optical properties of metal nanoparticles: the influence of size, shape, and dielectric environment. J Phys Chem B. 2003;107(3):668-77.

38. Rivas L, Sanchez-Cortes S, García-Ramos JV, Morcillo G. Growth of silver colloidal particles obtained by citrate reduction to increase the raman enhancement factor. Langmuir. 2001;17(3):574-7.

39. Stamplecoskie KG, Scaiano JC. Light emitting diode irradiation can control the morphology and optical properties of silver nanoparticles. J Am Chem Soc. 2010;132(6):1825-7.

40. Mack D, Davies A, Harris L, Jeeves R, Pascoe B, Knobloch JM, Rohde H, Wilkinson T. Staphylococcus epidermidis in biomaterial-associated infections. In: Moriarty TF, Zaat SAJ, Busscher HJ, editors. Biomaterials Associated Infection: Springer New York; 2013. p. 25-56.

41. Sievert DM, Ricks P, Edwards JR, Schneider A, Patel J, Srinivasan A, Kallen A, Limbago B, Fridkin S. Antimicrobial-resistant pathogens associated with healthcare-associated infections: summary of data reported to the National Healthcare Safety Network at the Centers for Disease Control and Prevention, 2009-2010. Infect Control Hosp Epidemiol. 2013;34(1):1-14.

42. Köck R, Becker K, Cookson B, van Gemert-Pijnen J, Harbarth S, Kluytmans J, Mielke M, Peters G, Skov R, Struelens M. Methicillin-resistant Staphylococcus aureus (MRSA): burden of disease and control challenges in Europe. 2010.

43. Gorwitz RJ. A review of community-associated methicillin-resistant Staphylococcus aureus skin and soft tissue infections. Pediatr Infect Dis J. 2008;27(1):1-7.

44. Hamerli P, Weigel T, Groth T, Paul D. Enhanced tissue-compatibility of polyethylenterephtalat membranes by plasma aminofunctionalisation. Surface Coating Technol. 2003;174-175(0):574-8.

45. Chernousova S, Epple M. Silver as antibacterial agent: ion, nanoparticle, and metal. Angewandte Chemie International Edition. 2013;52(6):1636-53.

46. Schierholz JM, Beuth J. Implant infections: a haven for opportunistic bacteria. J Hosp Infect. 2001;49(2):87-93.

47. Zhao L, Wang H, Huo K, Cui L, Zhang W, Ni H, Zhang Y, Wu Z, Chu PK. Antibacterial nano- structured titania coating incorporated with silver nanoparticles. Biomaterials. 2011;32(24):5706-16. 
